\title{
Combined AFM and confocal fluorescence microscope for applications in bio-nanotechnology
}

\author{
R. KASSIES*, K. O. VAN DER WERF †, A. LENFERINK†, C. N. \\ HUNTER + , J. D. OLSEN + , V. SUBRAMANIAM*† \& C. OTTO*† \\ *BMTI Institute for Biomedical Technology, and $\dagger M E S A+$ Institute for Nanotechnology, Faculty of \\ Science and Technology, University of Twente, PO Box 217, 7500 AE Enschede, The Netherlands \\ $\ddagger$ Robert Hill Institute for Photosynthesis Research and Krebs Institute for Biomolecular Research, \\ Department of Molecular Biology and Biotechnology, University of Sheffield, Western Bank, Sheffield \\ S1O 2TN, U.K.
}

Key words. AFM, confocal fluorescence microscopy, combined microscope, nano-manipulation, simultaneous operation.

\section{Summary}

We present a custom-designed atomic force fluorescence microscope (AFFM), which can perform simultaneous optical and topographic measurements with single molecule sensitivity throughout the whole visible to near-infrared spectral region. Integration of atomic force microscopy (AFM) and confocal fluorescence microscopy combines the high-resolution topographical imaging of AFM with the reliable (bio)-chemical identification capability of optical methods. The AFFM is equipped with a spectrograph enabling combined topographic and fluorescence spectral imaging, which significantly enhances discrimination of spectroscopically distinct objects. The modular design allows easy switching between different modes of operation such as tip-scanning, sample-scanning or mechanical manipulation, all of which are combined with synchronous optical detection. We demonstrate that coupling the AFM with the fluorescence microscope does not compromise its ability to image with a high spatial resolution. Examples of several modes of operation of the AFFM are shown using two-dimensional crystals and membranes containing lightharvesting complexes from the photosynthetic bacterium Rhodobacter sphaeroides.

\section{Introduction}

Atomic force microscopy (AFM) (Binnig et al., 1986) has evolved into a powerful tool in biological research. It has the unique ability to provide structural information on the (sub)nanometre scale in a biologically relevant aqueous environment (Hansma et al., 1994; Bustamante et al., 1997). Detailed images of single protein structures have appeared in

Correspondence to: Dr Cees Otto. Tel.: +31 (0)53 489 3157; fax: +31 (0)53489 1105; e-mail: c.otto@tnw.utwente.nl the literature (Czajkowsky \& Shao, 1998; Viani et al., 2000), although most high-resolution structural information is obtained on crystallized protein samples (Muller et al., 1999b; Stahlberg et al., 2001; Scheuring et al., 2003; Bahatyrova et al., 2004b; Fotiadis et al., 2004b). Measurements on membrane proteins in their native membranes (Fotiadis et al., 2004a; Bahatyrova et al., 2004a; Scheuring et al., 2004) provide information on the natural higher order organization of these proteins. In addition to being an imaging tool, the AFM can be used to measure mechanical properties of proteins and the strength of inter- and intramolecular bonds (Lee et al., 1994; Hinterdorfer et al., 1996; Rief et al., 1997; Muller et al., 1999a; Oesterhelt et al., 2000). Furthermore, using the AFM tip as a manipulation tool allows the precise and controlled modification of biological systems from the level of cells down to the scale of individual molecules (Schoenenberger \& Hoh, 1994; Thalhammer et al., 1997; Fotiadis et al., 2002).

Traditional AFM imaging is based on very general tipsample interactions, which makes the technique applicable to a wide range of samples. However, this also strongly limits its capacity to identify different objects comprising the sample, unless they have a very distinct shape or size. Several other modes of AFM, such as lateral force microscopy (Mate et al., 1987; Marti et al., 1990; Overney et al., 1994), chemical force microscopy (Frisbie et al., 1994; McKendry et al., 1998; Wong et al., 1998) and phase contrast imaging (Anczykowski et al., 1996; Cleveland et al., 1998; Noy et al., 1998), use materialspecific tip-sample interactions to improve the chemical specificity. However, these modes are also often not distinctive enough to identify multiple surface species reliably.

Optical spectroscopy and imaging, by contrast, are wellestablished techniques enabling the spectroscopic discrimination of distinct species in the sample. By fluorescent labelling of specific proteins it is possible to follow the processes and dynamics of these components within living cells. The advent 
of more sensitive detectors also enables the measurement of fluorescence from individual molecules (Weiss, 1999). Optical parameters such as intensity, wavelength, polarization and fluorescence lifetime provide valuable information about the specimen and its surroundings. However, one of the major disadvantages of optical spectroscopy is its comparatively poor spatial resolution, limited by diffraction to about half the wavelength of the light used.

The strengths and weaknesses of both techniques complement each other. Therefore, a combination of AFM and optical spectroscopy would provide a very powerful tool in biological research. The ultimate goal of such a combined AFM and fluorescence microscope (for which we propose the acronym AFFM) must be the simultaneous recording of both a high-resolution structural image as well as a chemically specific optical image.

A combination of topographic and optical imaging is also realized in near-field scanning optical microscopy (NSOM) (Dunn, 1999). In this technique, a tapered optical fibre with a subwavelength-sized aperture (typically $70-120 \mathrm{~nm}$ ) is scanned near the sample surface $(<10 \mathrm{~nm})$. The strongly localized optical near field at the aperture illuminates the sample, resulting in an optical image with a spatial resolution determined by the aperture size. During scanning the contours of the sample surface are followed by the fibre tip, yielding a topographic image of the same area. However, in order to achieve sufficient optical throughput, the aperture size has a lower limit, which leads to a much lower topographic resolution than that achievable with AFM. In NSOM, the same probe is used to obtain both topographic and optical images, which can lead to artefacts in the optical signal caused by the $z$ motion of the near-field probe (Hecht et al., 1997). An additional limitation of NSOM is its restriction to surface imaging, in contrast to confocal microscopy, which enables optical sectioning and three-dimensional (3-D) imaging. Furthermore, the use of a fragile optical probe complicates the operation of the microscope. Integration of AFM and confocal fluorescence microscopy provides an easier and more flexible way of combining high topographic resolution with sensitive spectroscopic identification capability.

Several examples of AFM and fluorescence microscope combinations have been reported in the literature over the last few years (Lieberman et al., 1996; Lal \& Proksch, 1997; Foubert et al., 2000; Horton et al., 2000; Kolodny et al., 2001; Noy \& Huser, 2003; Gradinaru et al., 2004). One of the most significant problems in these designs is the optical background in the fluorescence detection caused by the laser used in the AFM to detect the cantilever position (Meyer \& Amer, 1988). As a result, most combined microscopes perform the AFM and fluorescence measurements sequentially. For simultaneous imaging, this laser light must be filtered from the detection path using high-quality filters, consequently blocking a potentially useful part of the spectrum (Gradinaru et al., 2004). An alternative approach is demonstrated by Noy \& Huser (2003), who implemented an interleaved scanning mode, in which each line is scanned twice during imaging. In the first scan the AFM collects the topographical information. During the second scan, the AFM laser is switched off and the optical information is collected. In this method, the two microscopes still operate sequentially on the same spot, and therefore cannot be used to perform mechanical manipulations on the sample while simultaneously recording optical responses.

Here we describe a custom-designed AFFM set-up that can perform truly simultaneous AFM and fluorescence imaging, force extension measurements and nano-manipulation. The instrument allows the use of any fluorescent dye throughout the whole visible to near-infrared (IR) spectral region with single molecule optical and mechanical sensitivity. In addition, we have incorporated a spectral fluorescence imaging capability in our combined microscope, which greatly enhances the (bio-)chemical identification capacity.

We demonstrate the AFFM on 2-D crystals and membranes containing LH1 and LH2 light-harvesting pigment protein complexes from the photosynthetic bacterium Rhodobacter sphaeroides. The spectral imaging mode is used to discriminate LH1 and LH2 in a mixed membrane sample, based on the difference between their fluorescence spectra (LH1 emission peak $\sim 890 \mathrm{~nm}, \mathrm{LH} 2 \sim 870 \mathrm{~nm})$.

\section{Design of the AFFM}

The combined microscope is based on a custom-designed stand-alone AFM (Van der Werf et al., 1993) and inverted confocal fluorescence microscope with single molecule sensitivity (Fig. 1). The complete AFFM system is placed on a vibrationisolated table. A sample scanning piezo stage (P-730 XY, Physik Instrumente, Germany) with a scan range of $40 \times 40 \mu \mathrm{m}^{2}$ and a minimal step size of $0.5 \mathrm{~nm}$ in closed loop mode is used by both microscopes. Additionally, the AFM tip can be controlled in 3D space by a piezo tube scanner, so that the AFM retains its full stand-alone functionality.

The confocal microscope uses a diode laser (Roithner Laser Technik, RLT80010MG, $\lambda=800 \mathrm{~nm}$ ) as a light source. In addition, all available laser lines in the visible wavelength range from an argon-krypton laser can be coupled to the instrument through a fibre connection. The laser beam is reflected via a dichroic beam splitter (Chroma, Q850LPXXR) towards an oil-immersion objective (Nikon, Plan Fluor 100×, NA 1.3), which focuses the light onto the sample. The fluorescence light is collected by the same objective and passes through the dichroic beam splitter. By switching a foldable mirror, the fluorescence light can be directed either towards a single photon counting avalanche photodiode (APD) (SPCMAQR-14, Perkin Elmer Optoelectronics) or towards a customdesigned prism-based spectrograph, with single molecule sensitivity, equipped with a liquid nitrogen-cooled CCD camera (Spec-10:100B, Princeton Instruments). Any remaining excitation light is removed from the detection path by a holographic notch filter (HNPF-800, Kaiser Optical Systems). The 
Fig. 1. Schematic layout of the AFFM with the controllers and computer interface cards. A 1050$\mathrm{nm}$-wavelength laser diode is placed inside the AFM head together with a narrow bandpass filter. In the confocal microscope, a dichroic beam splitter (BS) reflects the excitation beam towards the objective. Collected fluorescence light passes this beam splitter towards the detectors. A notch filter (NF) and an AFM laser suppression filter (ASF) remove the backscattered excitation light and collected AFM laser light from the detection path. By switching the foldable mirror $(\mathrm{M})$, the fluorescence light can be directed towards the APD or the spectrograph.

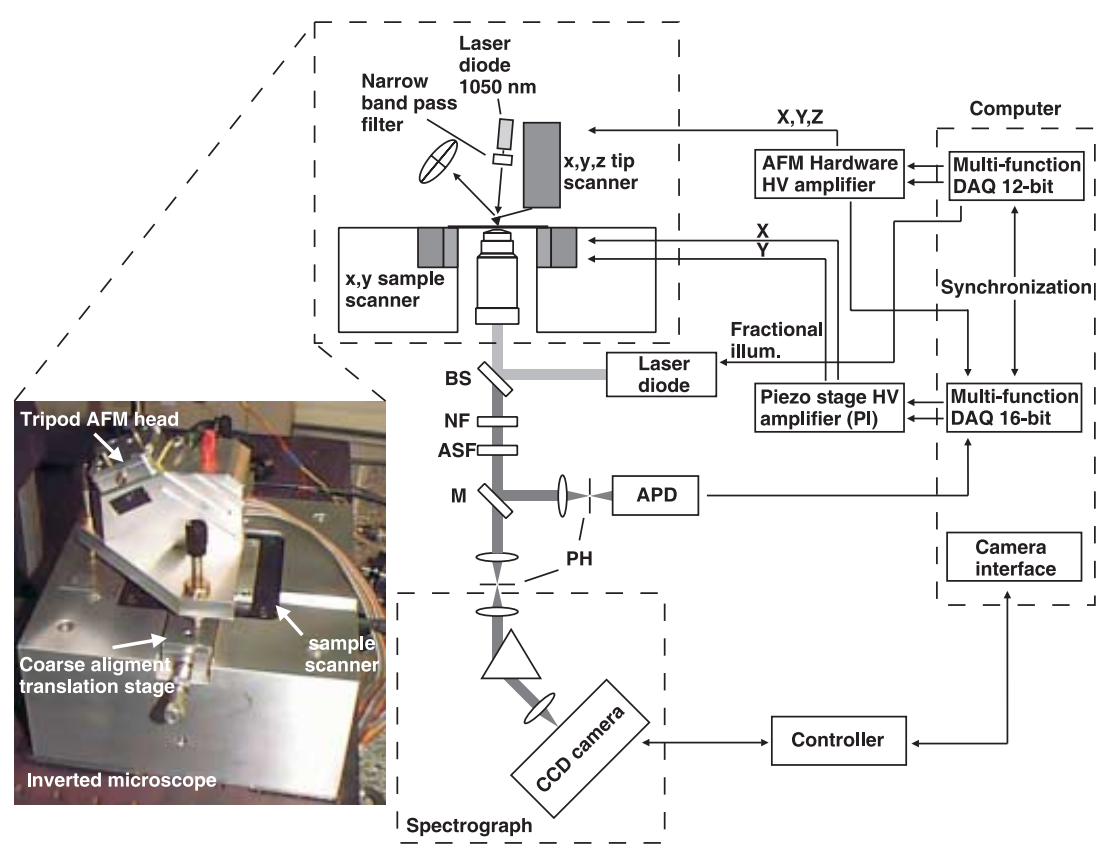

APD is suitable for measurements that require high time resolution, such as recording fast dynamics in fluorescence timetraces or rapid fluorescence imaging. The spectrographCCD camera combination can be used for conventional spectral imaging or for recording spectral timetraces, where a complete spectrum is recorded for each image pixel or time step, respectively.

In AFM systems using optical beam deflection as a sensing method, part of this laser light is transmitted through and passes beside the cantilever. In combination with a fluorescence microscope, this light is collected by the objective and enters the detection path, creating a significant background in the fluorescence measurements. Furthermore, this light may easily excite the fluorophores in the sample. This can cause an unwanted photobleaching of the fluorescent material. To prevent these problems in the AFFM, a diode laser with a wavelength of $1050 \mathrm{~nm}$ (Roithner Laser Technik, RLT106010MG) was installed. A similar approach was taken by Ebenstein $e$ al. (2002). In addition, we positioned a narrow bandpass filter (CVI Laser, F10-1050-4) inside the AFM head (Fig. 1), which only transmits the 1050-nm emission peak and suppresses the sidebands typical of diode laser emission. The advantage of using this laser wavelength in combination with the bandpass filter is two-fold. First, it allows the use of the AFM in combination with essentially all fluorophores in the visible to near-IR spectrum, without the AFM laser exciting the fluorescent molecules or overlapping with their emission spectra. Second, the narrow wavelength range, limited by the bandpass filter, makes it possible to effectively remove this light from the detection path of the fluorescence microscope by a suitable notch or lowpass filter. This allows single molecule fluorescence detection extended to emission wavelengths in the near-IR during operation of the AFM. Imaging and performing force interactions with the sample while simultaneously recording optical responses is now possible.

Another common problem compromising AFM measurements is interference and optical feedback effects due to AFM laser light scattered from the reflective sample and objective surfaces. We implemented a recently developed high-frequency laser current modulation technique to circumvent this problem (Kassies et al., 2004).

The sample scanner is embedded in the solid support of the inverted microscope (Fig. 1). The AFM tripod is equipped with a wide base plate, which effectively spans this scanner. Two legs of the AFM head are placed on two perpendicularly orientated translation stages, which allows the coarse positioning of the AFM tip with respect to the optical axis of the confocal microscope. Fine adjustment of the tip position can be made using the piezo tube inside the AFM head.

The sample scanner and the tip scanner (piezo tube) are addressed independently, which allows the flexible movement of the sample and the tip relative to the excitation volume and relative to each other. It also provides the possibility to switch easily between different modes of operation, such as sample scanning, tip scanning or mechanical manipulation (Fig. 2). The computer controls the sample scanner through a 16-bit multifunction data acquisition card (National Instruments, 6052E). The 16-bit resolution makes it possible to scan the sample over the full range of $40 \times 40 \mu \mathrm{m}^{2}$ with a minimum step size of $<0.5 \mathrm{~nm}$. The tip scanner, which has a maximum scan range of $3 \times 3 \mu \mathrm{m}^{2}$, is controlled through a 12-bit multifunction data acquisition card (National Instruments, MIO16-E4). A custom Labview program (National Instruments) is used to control the AFFM. 


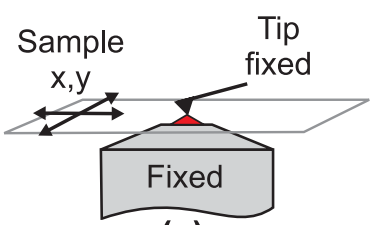

(a)

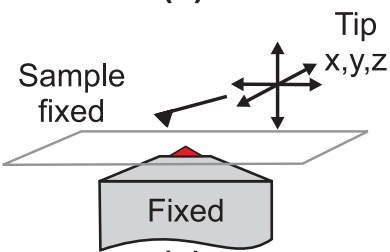

(c)

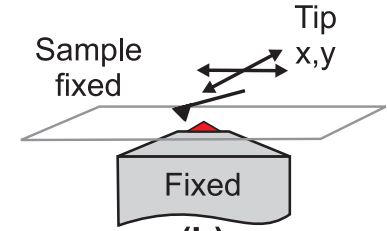

(b)

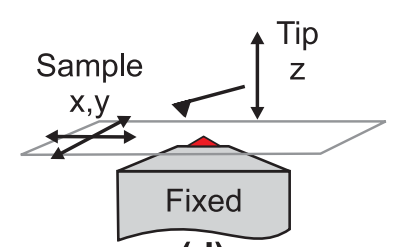

(d)
Fig. 2. Different modes of operation of the AFFM: (a) simultaneous imaging by scanning the sample between a fixed tip and a fixed focus; (b) AFM imaging by lateral tip scanning with a specific area (selected by positioning the sample stage) in the excitation volume; (c) force-extension imaging with the tip moving in three dimensions; (d) force-extension imaging with the tip ramping perpendicular to the surface and the sample moving in lateral directions. In modes (c) and (d) optical timetraces are recorded and stored along with the force-extension curves.

\section{Performance of the AFM}

The combination of the AFM with the fluorescence microscope does not compromise the ability of the AFM to image with a high spatial resolution. This is demonstrated in Fig. 3, which shows high-resolution images of 2-D crystals of the membrane proteins LH2 and LH1, isolated from the bacterium R. sphaeroides (Bahatyrova et al., 2004b). These images were measured in tapping mode (Veeco Instruments, MSCT-AUHW, $\mathrm{Si}_{3} \mathrm{~N}_{4}$ tip F, $k=0.5 \mathrm{~N} \mathrm{~m}^{-1}$ ) in buffer solution (10 mm HEPES, pH 7.5, $150 \mathrm{~mm} \mathrm{KCl).} \mathrm{The} \mathrm{crystals} \mathrm{were}$ immobilized on a mica substrate. The AFM images were obtained by scanning the sample, using the internal position feedback of the sample stage, which prevents any

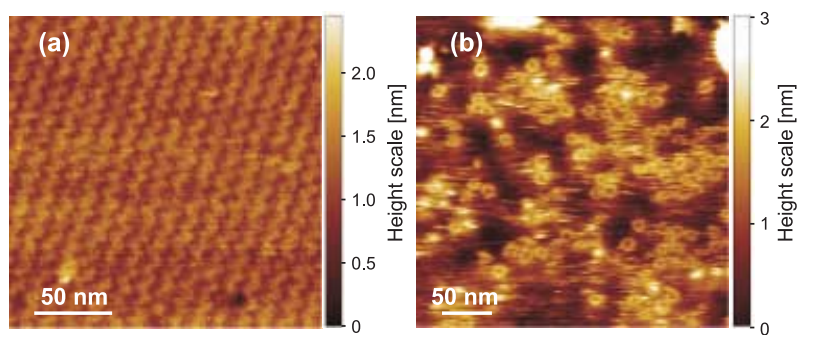

Fig. 3. High-resolution topographic images measured with the AFFM. (a) Image of a 2-D crystal of LH2 complexes. The individual LH2 complexes are packed in a zig-zag pattern, which can be clearly resolved by the AFM. (b) Image of a 2-D crystal of LH1 complexes. The ring-shaped LH1 complexes with a diameter of $\sim 11 \mathrm{~nm}$ are slightly larger than the LH2 complexes. The ring shape of these proteins can clearly be resolved.

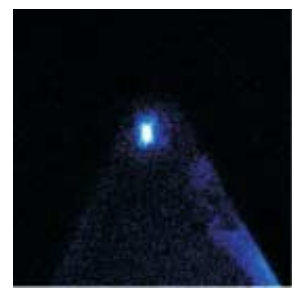

(a)

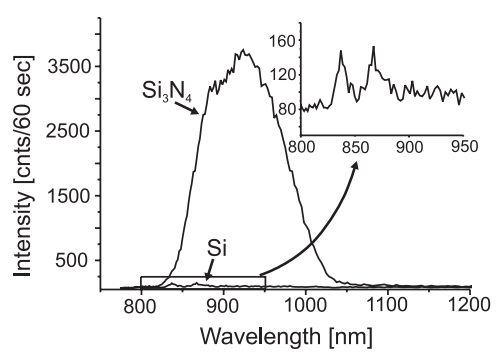

(b)
Fig. 4. (a) Luminescence image of an $\mathrm{Si}_{3} \mathrm{~N}_{4}$ cantilever, with the tip clearly visible. (b) Luminescence spectrum of $\mathrm{Si}$ and $\mathrm{Si}_{3} \mathrm{~N}_{4}$ tips measured by the spectrograph. Excitation intensity $=7 \mu \mathrm{W}$; emission accumulation time $=1 \mathrm{~min}$. The background level of $\sim 80$ counts is the intrinsic offset of the CCD camera.

image distortions due to non-linearities in the scanning motion.

\section{Luminescence of $\mathrm{Si}_{3} \mathrm{~N}_{4}$ and $\mathrm{Si}$ tips}

A direct spatial correlation between topographic and optical features is obtained when the AFM tip is aligned within the excitation focus of the confocal microscope. We observed that proper alignment inevitably led to luminescence caused by the tip material itself, in addition to the direct scattering of light from the tip. This was primarily the case with $\mathrm{Si}_{3} \mathrm{~N}_{4}$ tips. The emission spectra of an $\mathrm{Si}_{3} \mathrm{~N}_{4}$ tip (Veeco Instruments, MSCTAUHW) and an Si tip (Mikromasch, NSC36/Cr-AuBS) excited with the 800-nm wavelength from the laser diode, were measured using the spectrograph and are presented in Fig. 4. The spectra were accumulated over a 1-min interval, using $7 \mu \mathrm{W}$ of laser light focused on the tip.

The luminescence from the $\mathrm{Si}_{3} \mathrm{~N}_{4}$ tip covers a broad spectral region and may spectrally overlap with the emission of the chromophores in the sample, in which case it cannot be removed by spectral filtering. The intensity of this luminescence is similar to the fluorescence intensity of a few LH1 or LH2 molecules. The Si tip, however, shows a different type of emission with much lower intensity (Fig. 4), which is negligible compared with typical single molecule emission intensities.

Crystalline silicon has an indirect band gap, and is therefore incapable of light emission. However, amorphous silicon and amorphous silicon nitride are known to be strongly photoluminescent (Wolford et al., 1983; Poolton \& Cros, 1991; Aydinli et al., 1996). Silicon nitride tips are generally manufactured from amorphous material, produced by low-pressure chemical vapour deposition (LPCVD) (Veeco Instruments, pers. comm.), and thus display considerable luminescence. Silicon tips, by contrast, are produced from crystalline silicon, explaining the low amount of luminescence produced by these tips. We conclude that it is imperative to use tips made from crystalline silicon or other low-luminescent materials during simultaneous AFM/optical measurements. 

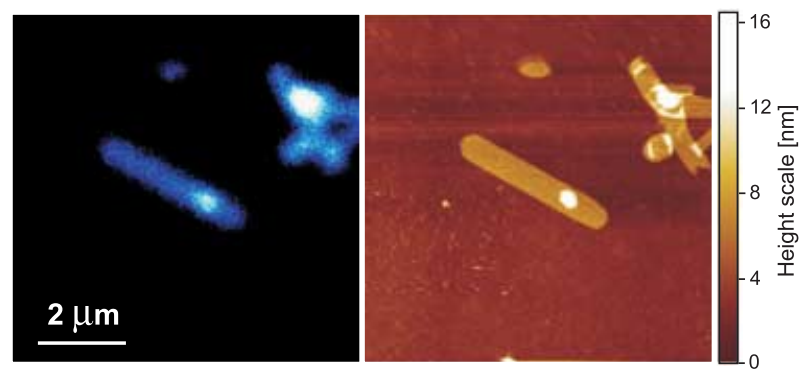

Fig. 5. Combined simultaneous imaging on 2-D LH2 crystals. The AFM tip was aligned with the optical axis of the fluorescence microscope. While scanning the sample, the height profile and optical information was detected simultaneously. The APD was used for the optical detection.

\section{Simultaneous topographic and optical imaging}

In simultaneous fluorescence and topographic imaging mode the sample is scanned between the microscope objective and the AFM tip. The vertical motion of the tip relative to the sample is induced by the piezo-tube inside the AFM in order to maintain a constant deflection. Fluorescence and topographical information are collected simultaneously and are inherently spatially correlated, owing to the coincidence of the excitation focus and the AFM tip.

Figure 5 shows simultaneously recorded fluorescence and topographic AFFM images of 2-D crystals of light-harvesting complexes LH2. The crystals were adsorbed on a poly-L-lysine$(0.01 \%)$ coated coverglass. The glasses were extensively cleaned in a solution of $65 \% \mathrm{HNO}_{3}$ before adding the coating. The measurement was performed in a solution of 50 mMTRIS, $\mathrm{pH} 7.8,150 \mathrm{~mm} \mathrm{KCl}$ in tapping mode using an Si tip (Mikromasch, NSC36/Cr-AuBS, $k=0.6 \mathrm{~N} \mathrm{~m}^{-1}$ ). The optical signal was recorded using the APD. The sample was scanned at $256 \times 256$ pixels with a pixel frequency of $2000 \mathrm{~Hz}$ and hence a recording time of the optical signal of $0.5 \mathrm{~ms}$ per pixel.

The LH2 crystals are observed as sheets with a length of several micrometres and a width up to $\sim 1 \mu \mathrm{m}$. The crystals are $\sim 6 \mathrm{~nm}$ high. A clear correlation exists between the topographic and optical images. Higher topographic features on the crystals correspond to a high amplitude of the fluorescence emission, indicating that a second layer of crystal is present rather than some impurity.

Confocal microscopy has a relatively low spatial resolution (limited by diffraction) compared with AFM. Therefore, AFM images usually contain more pixels per unit area than confocal microscopy images. In a combined AFFM measurement the number of pixels per unit area should be high enough to reveal topographical details. Consequently, the optical image is strongly over-sampled and the continuous illumination of the sample over many pixels may cause rapid photobleaching. A decrease in excitation intensity can reduce photobleaching, but can also lead to a significant decrease in signal-to-noise ratio in the optical image. This is particularly important in single

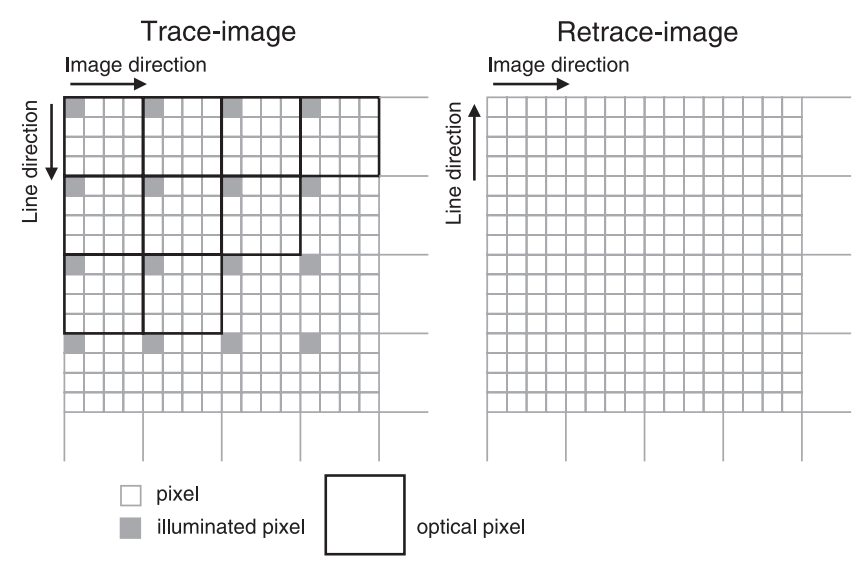

Fig. 6. Fractional illumination can be applied in simultaneous imaging to reduce photobleaching. The total illumination time is reduced by illuminating the sample only for a small fraction of the scanned pixels during the 'trace-image' and by switching the laser off during the 'retrace-image'. After the measurement, the square sections containing one illuminated pixel can be binned to form a single optical pixel.

molecule experiments where the fluorescence intensity is low. The over-sampling of the optical image allows a different approach to reduce bleaching without a decrease in signal-tonoise ratio. The total illumination time can be minimized by switching the excitation laser on only during a small fraction of the scanned pixels in the 'trace-image', and leaving the laser off during the 'retrace-image'. To achieve this, a TTL-pulse pattern is generated together with the $x, y$ scan pattern, marking the pixels where the laser should be on. This pattern is synchronously sent to the laser diode controller during scanning. The same pattern can be sent as a gating signal to the APD. After the measurement, the square sections containing one illuminated pixel can be binned to form a single optical pixel. This is illustrated in Fig. 6 where, as an example, one in every four scanned pixels is illuminated in the line direction as well as in the image direction. The ratio between the number of scanned pixels and illuminated pixels can be set to any value. In the particular example of Fig. 6 the illumination time is reduced by a factor of 32 .

\section{Combined AFM and spectral fluorescence imaging}

The ability to perform spectral fluorescence imaging strongly enhances the chemical identification capacity of the instrument. In conventional spectral imaging mode, a complete fluorescence spectrum is measured for each pixel. Multiple fluorescent components can be easily distinguished even when their emission spectra partially overlap. In order to obtain a spectrum with a sufficient signal-to-noise ratio, the acquisition time per pixel is usually of the order of $10 \mathrm{~ms}$ or more. In AFM imaging the time per pixel is usually much lower, typically ranging from 0.2 to $0.5 \mathrm{~ms}$. Because of this discrepancy, we record AFM and spectral images sequentially. 

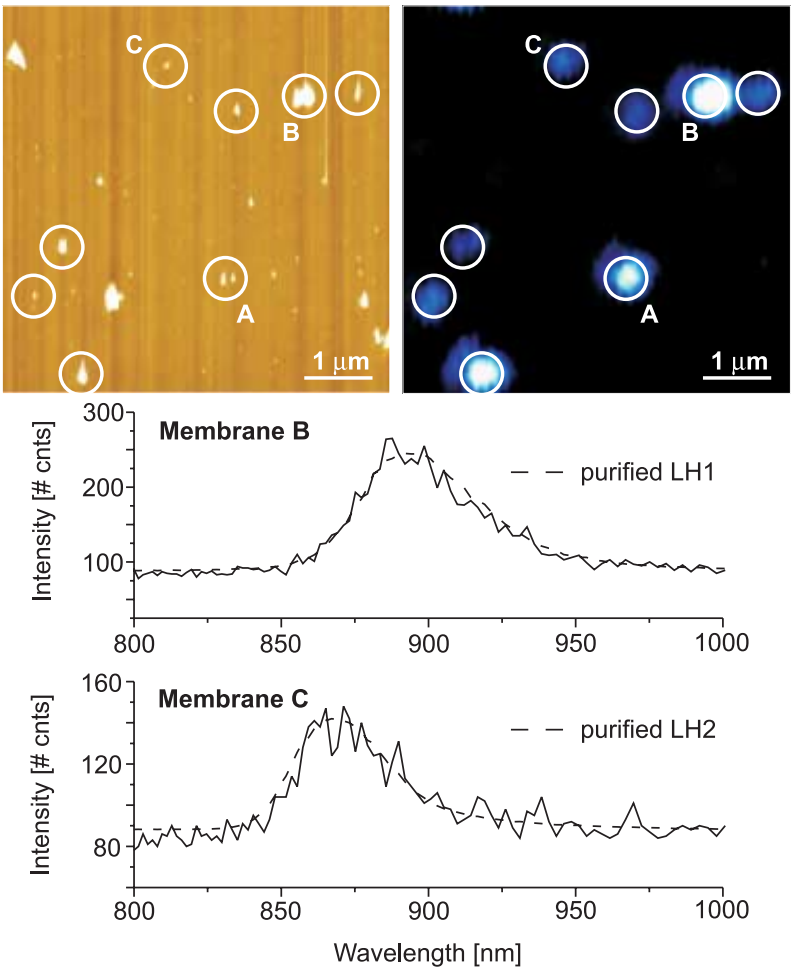

Fig. 7. Combined AFM and fluorescence spectral imaging on membranes fragments from Rhodobacter sphaeroides, containing both LH1 and LH2 complexes. First, a fluorescence spectral image is acquired, followed by an AFM image on the same area. Membranes containing light-harvesting complexes can be distinguished from other surface features by comparing the optical and topographic images. In the spot marked by $\mathrm{A}$, two membrane fragments lying close together cannot be resolved in the optical image, demonstrating the superior lateral resolution of the AFM. The content of the membrane fragments can be estimated from the spectral information. The membrane marked by B shows a spectrum closely resembling the emission spectrum of LH1, whereas the spectrum of the membrane marked by C closely resembles that of LH2. The solid lines represent spectra from a single pixel in each membrane fragment. For comparison, the measured emission spectra of concentrated purified solutions of LH1 and LH2 were scaled and plotted in dashed lines along with the membrane spectra.

The potential of combined AFM and spectral imaging is demonstrated in Fig. 7. In this measurement, small membrane fragments of the photosynthetic bacterium R. sphaeroides were immobilized on a coverglass coated with poly-L-lysine. The membranes contain both types of light-harvesting complexes, LH1 and LH2. The sample was measured in a buffer solution containing $10 \mathrm{~mm}$ HEPES, pH 7.5, $150 \mathrm{~mm} \mathrm{KCl}$. The spectral image was recorded on an area of $5.5 \times 5.5 \mu \mathrm{m}^{2}$ at $128 \times 128$ pixels and an integration time of $35 \mathrm{~ms}$ per pixel. Subsequently, a tapping mode AFM image was measured on the same area using an $\mathrm{Si}_{3} \mathrm{~N}_{4}$ tip (Veeco Instruments, MSCTAUHW, $k=0.5 \mathrm{~N} \mathrm{~m}^{-1}$ ) at $512 \times 512$ pixels. The fragments are $50-100 \mathrm{~nm}$ in diameter and $7-50 \mathrm{~nm}$ in height.
The superior lateral resolution of the AFM can be clearly seen at location A in the images. Here, two separate membrane fragments are observed lying close together in the AFM image, but cannot be resolved in the optical image. By contrast, the fluorescence measurement identifies those topographic structures that contain varying amounts of lightharvesting complexes. The optical spots clearly co-localize with topographic features, although there are also objects in the AFM image that are absent in the optical image. These non-fluorescent objects could well be membrane fragments that do not contain any LH complexes. Moreover, the spectral data collected on each pixel provide information about the composition of the different membrane fragments. The spectrum emitted by membrane B (Fig. 7) closely resembles the LH1 emission spectrum, suggesting that this membrane mainly contains LH1 complexes. By contrast, membrane C shows a spectrum resembling LH2 emission, suggesting a larger number of LH2 complexes in this membrane. For comparison, the measured emission spectra of concentrated solutions of purified LH1 and LH2 complexes were scaled and plotted along with the membrane spectra.

\section{Combined optical and force extension measurements/ nanomanipulation}

The desirability of combined force spectroscopy and single molecule fluorescence measurements has been addressed by Wallace et al. (2003) and Weiss (1999). For the measurement of mechanical properties of objects, interaction forces and adhesion forces, the so-called force-extension mode is often used. In this mode, the AFM tip is lowered towards the surface until it makes contact and a certain preset force is reached, after which the tip is retracted. In this way, a force-extension curve is measured and stored for each image pixel. In our AFFM a fluorescence timetrace is recorded by the APD and stored along with the force-extension curve for each pixel. This type of imaging can either be done with the sample in a fixed location and the tip moving in three dimensions (Fig. 2c), or with a laterally scanning sample and the tip only ramping up and down (Fig. 2d). In general, this mode provides optical information from the sample as a function of the 3-D position of the tip.

In a force-extension measurement, the maximum force that the tip exerts on the sample is well controlled, and the optical response of a molecular system can be observed as a function of the applied force.

The combined force-extension mode (Fig. 2c) is illustrated using the collection of the luminescence signal of a silicon nitride tip as a function of the 3-D tip position (Fig. 8). The excitation light was focused on the top layer of a clean coverglass. The $\mathrm{Si}_{3} \mathrm{~N}_{4}$ tip was ramped perpendicular to the glass surface with a frequency of $1 \mathrm{~Hz}$ in liquid. At each pixel, a complete force-extension curve was recorded with simultaneous detection of the luminescence of the $\mathrm{Si}_{3} \mathrm{~N}_{4}$ tip material in 


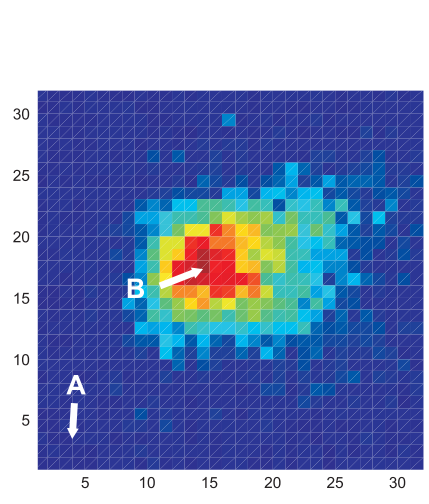

(a)

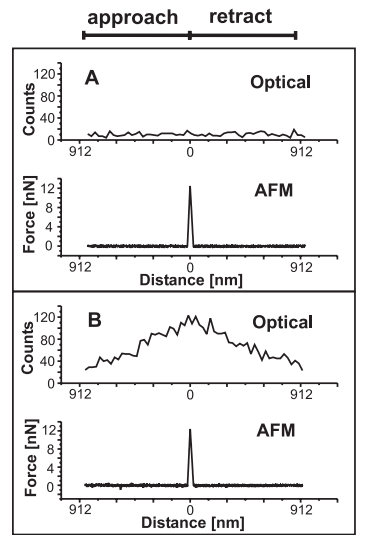

(b)
Fig. 8. Combined force-extension measurement, where a complete force-extension curve and optical trace is collected for each lateral pixel. The total lateral range is $2.7 \times 2.7 \mu \mathrm{m}^{2}$. The force-extension curves were collected over a vertical range of $\sim 900 \mathrm{~nm}$. (a) Image reconstructed from the optical traces. In each pixel the number of photon counts at the moment when the tip touched the surface was plotted. When the $\mathrm{Si}_{3} \mathrm{~N}_{4}$ tip enters the focus, the optical signal increases. (b) Two examples of the total data-set. In location A, where the tip approaches outside the focus, no optical signal is recorded. In location B the tip approaches in the centre of the focus, resulting in a clear fluorescence signal from the tip material.

the optical timetraces. It follows that the maximum luminescence intensity is detected when the tip touches the glass surface. The measurement was made on $32 \times 32$ pixels over a total lateral range of $2.7 \times 2.7 \mu \mathrm{m}^{2}$ and a vertical range of $\sim 900 \mathrm{~nm}$. The force-extension curve and the optical trace were sampled 5000 times, yielding a time resolution of $0.2 \mathrm{~ms}$. The optical data were then binned 100 times. The image of Fig. 8(a) was obtained from the optical traces. The amplitude in the image reflects the number of counts at the moment when the tip touched the surface and reached maximum deflection. The force-extension curve and the corresponding timetrace of two particular pixels are shown in Fig. 8(b). In pixel A the tip touches the glass outside the focus and therefore the fluorescence signal is unchanged during the force-extension curve. In pixel B, the tip is lowered in the centre of the focus and the fluorescence signal increases until the tip touches the surface.

Figure 8 demonstrates the possibility of collecting optical information as a function of the tip position. More importantly, in the regime of the force-extension curve where the tip touches the sample, the data set provides optical information as a function of the well-controlled interaction force.

\section{Conclusions}

The integration of an AFM and a confocal fluorescence microscope provides detailed structural information about the specimen with high (bio)-chemical specificity. The combined information acquired by the AFFM allows the spectroscopic identification of topographic features.

The AFFM presented here can perform simultaneous topographic and optical imaging with single molecule sensitivity. By shifting the AFM laser to a wavelength of $1050 \mathrm{~nm}$, chromophores that have excitation and emission wavelengths anywhere in the visible to near-IR region can be used. Application of fractional illumination of the sample during simultaneous imaging allows high-resolution topographic mapping without rapid photobleaching of the chromophores. We have demonstrated that coupling the AFM with the fluorescence microscope does not compromise high-resolution imaging with the AFFM. The incorporation of fluorescence spectral imaging strongly enhances the chemical identification capability of the system.

The possibility of addressing the sample scanner and the tip scanner independently, synchronized with the optical detection, results in a very flexible instrument, allowing many different types of experiments. The combined optical and force-extension mode provides the possibility of measuring optical responses of the specimen as a function of a well-controlled force.

These multiple imaging modes and the flexibility and modularity of the AFFM make it a powerful tool for bionanotechnology applications.

\section{Acknowledgements}

This work was supported by the Netherlands Organization for Scientific Research NWO. We would like to thank Ing. Frans Segerink for software support.

\section{References}

Aydinli, A., Serpengüzel, A. \& Vardar, D. (1996) Visible photoluminescence from low temperature deposited hydrogenated amorphous silicon nitride. Solid State Commun. 98, 273-277.

Anczykowski, B., Kruger, D., Bancock, K.L. \& Fuchs, H. (1996) Basic properties of dynamic force spectroscopy with the scanning force microscope in experiment and simulation. Ultramicroscopy, 66, 251-259.

Bahatyrova, S., Frese, R.N., Siebert, C.A., Olsen, J.D., Van der Werf, K.O., Van Grondelle, R., Niederman, R.A., Bullough, P.A., Otto, C. \& Hunter, C.N. (2004a) The native architecture of a photosynthetic membrane. Nature, 430, 1058-1062.

Bahatyrova, S., Frese, R.N., Van der Werf, K.O., Otto, C., Hunter, C.N. \& Olsen, J.D. (2004b) Flexibility and size heterogeneity of the LH1 light harvesting complex revealed by atomic force microscopy. J. Biol. Chem. 279, 21327-21333.

Binnig, G., Quate, C.F. \& Gerber, C. (1986) Atomic force microscope. Phys. Rev. Lett. 56, 930-933.

Bustamante, C., Rivetti, C. \& Keller, D.J. (1997) Scanning force microscopy under aqueous solutions. Curr. Opin. Struct. Biol. 7, 709-716.

Cleveland, J.P., Anczykowski, B., Schmid, A.E. \& Elings, V.B. (1998) Energy dissipation in tapping-mode atomic force microscopy. Appl. Phys. Lett. 72, 2613-2615.

Czajkowsky, D.M. \& Shao, Z. (1998) Submolecular resolution of single macromolecules with atomic force microscopy. FEBS Lett. 430, 51-54. 
Dunn, R.C. (1999) Near-field scanning optical microscopy. Chem. Rev. 99, 2891-2927.

Ebenstein, Y., Mokari, T. \& Banin, U. (2002) Fluorescence quantum yield of $\mathrm{CdSe} / \mathrm{ZnS}$ nanocrystals investigated by correlated atomic-force and single-particlefluorescence microscopy. Appl. Phys. Lett. 80, 4033-4035.

Fotiadis, D., Liang, Y., Filipek, S., Saperstein, D.A., Engel, A. \& Palczewski, K. (2004a) The G protein-coupled receptor rhodopsin in the native membrane. FEBS Lett. 564, 281-288.

Fotiadis, D., Qian, P., Philippsen, A., Bullough, P.A., Engel, A. \& Hunter, C.N. (2004b) Structural analysis of the reaction center light-harvesting complex I photosynthetic core complex of Rhodospirillum rubrum using atomic force microscopy. J. Biol. Chem. 279, 2063-2068.

Fotiadis, D., Scheuring, S., Muller, S.A., Engel, A. \& Muller, D.J. (2002) Imaging and manipulation of biological structures with the AFM. Micron, 33, 385-397.

Foubert, P., Vanoppen, P., Martin, M., Gensch, T., Hofkens, J., Helser, A., Seeger, A., Taylor, R.M., Rowan, A.E., Nolte, R.J.M. \& De Schrijver, F.C. (2000) Mechanical and optical manipulation of porphyrin rings at the submicrometer scale. Nanotechnology, 11, 16-23.

Frisbie, C.D., Rozsnyai, L.F., Noy, A., Wrighton, M.S. \& Lieber, C.M. (1994) Functional group imaging by chemical force microscopy. Science, 265 , 2071-2074.

Gradinaru, C.C., Martinsson, P., Aartsma, T.J. \& Schmidt, T. (2004) Simultaneous atomic force and two-photon fluorescence imaging of biological specimens in vivo. Ultramicroscopy, 99, 235-245.

Hansma, P.K., Cleveland, J.P., Radmacher, M., Walters, D.A., Hillner, P., Bezanilla, M., Fritz, M., Vie, D., Hansma, H.G., Prater, C.B., Massie, J., Fukunaga, L., Gurley, J. \& Elings, V. (1994) Tapping mode atomic force microscopy in liquids. Appl. Phys. Lett. 64, 1738-1740.

Hecht, B., Bielefeldt, H., Inouye, Y., Pohl, D.W. \& Novotny, L. (1997) Facts and artifacts in near-field optical microscopy. J. Appl. Phys. 81, 2492-2498.

Hinterdorfer, P., Baumgartner, W., Gruber, H.J., Schilcher, K. \& Schindler, H. (1996) Detection and localization of individual antibody-antigen recognition events by atomic force microscopy. Proc. Natl Acad. Sci. U.S.A., 93, 3477-3481.

Horton, M., Charras, G., Ballestrem, C. \& Lehenkari, P. (2000) Integration of atomic force and confocal microscopy. Single Mol. 1, 135-137.

Kassies, R., Van der Werf, K.O., Bennink, M.L. \& Otto, C. (2004) Removing optical feedback and interference artifacts in AFM measurements by application of high frequency laser current modulation. Rev. Sci. Instrum. 75, 689-693.

Kolodny, L.A., Willard, D.M., Carillo, L.L., Nelson, M.W. \& Van Orden, A. (2001) Spatially correlated fluorescence/AFM of individual nanosized particles and biomolecules. Anal. Chem. 73, 1959-1966.

Lal, R. \& Proksch, R. (1997) Multimodal atomic force microscopy: biological imaging using atomic force microscopy combined with light fluorescence and confocal microscopies and electrophysiologic recording. Int. J. Imag. Syst. Tech. 8, 293-300.

Lee, G.U., Kidwell, D.A. \& Colton, R.J. (1994) Sensing discrete streptavidinbiotin interactions with atomic force microscopy. Langmuir, 10, 354357.

Lieberman, K., Ben-Ami, N. \& Lewis, A. (1996) A fully integrated nearfield optical, far-field optical, and normal-force scanned probe microscope. Rev. Sci. Instrum. 67, 3567-3572.

Marti, O., Colchero, J. \& Mlynek, J. (1990) Combined scanning force and friction microscopy of mica. Nanotechnology, 1, 141-144.

Mate, C.M., McClelland, G.M., Erlandsson, R. \& Chiang, S. (1987) Atomic-scale friction of a tungsten tip on a graphite surface. Phys. Rev. Lett. 59, 1942-1945.
McKendry, R., Theoclitou, M.-E., Rayment, T. \& Abell, C. (1998) Chiral discrimination by chemical force microscopy. Nature, 391, 566-568.

Meyer, G. \& Amer, N.M. (1988) Novel approach to atomic force microscopy. Appl. Phys. Lett. 53, 1045-1047.

Muller, D.J., Baumeister, W. \& Engel, A. (1999a) Controlled unzipping of a bacterial surface layer with atomic force microscopy. Proc. Natl Acad. Sci. U.S.A., 96, 13170-13174.

Muller, D.J., Fotiadis, D., Scheuring, S., Muller, S.A. \& Engel, A. (1999b) Electrostatically balanced subnanometer imaging of biological specimens by atomic force microscope. Biophys. J. 76, 1101-1111.

Noy, A. \& Huser, T.R. (2003) Combined force and photonic probe microscope with single molecule sensitivity. Rev. Sci. Instrum. 74, 1217-1221.

Noy, A., Sanders, C.H., Vezenov, D.V., Wong, S.S. \& Lieber, C.M. (1998) Chemically-sensitive imaging in tapping mode by chemical force microscopy: relationship between phase lag and adhesion. Langmuir, 14, 1508-1511.

Oesterhelt, F., Oesterhelt, D., Pfeiffer, M., Engel, A., Gaub, H.E. \& Muller, D.J. (2000) Unfolding pathways of individual bacteriorhodopsins. Science, 288, 143-146.

Overney, R.M., Meyer, E., Frommer, J. \& Guntherodt, H.-J. (1994) Force microscopy study of friction and elastic compliance of phase-separated organic thin films. Langmuir, 10, 1281-1286.

Poolton, N.R.J. \& Cros, Y. (1991) Luminescence excitation and bleaching involving dangling bonds in silicon nitride. J. Phys. I France, 1, 1335-1345.

Rief, M., Gautel, M., Oesterhelt, F., Fernandez, J.M. \& Gaub, H.E. (1997) Reversible unfolding of individual titin immunoglobulin domains by AFM. Science, 276, 1109-1112.

Scheuring, S., Seguin, J., Marco, S., Levy, D., Breyton, C., Robert, B. \& Rigaud, J.L. (2003) AFM characterization of tilt and intrinsic flexibility of Rhodobacter sphaeroides light harvesting complex 2 (LH2). J. Mol. Biol. 325, 569-580.

Scheuring, S., Sturgis, J.N., Prima, V., Bernadac, A., Levi, D. \& Rigaud, J.-L. (2004) Watching the photosynthetic apparatus in native membranes. Proc. Natl Acad. Sci. U.S.A., 101, 11293-11297.

Schoenenberger, C.A. \& Hoh, J.H. (1994) Slow cellular dynamics in MDCK and R 5 cells monitored by time-lapse atomic force microscopy. Biophys. J. 67, 929-936.

Stahlberg, H., Muller, D.J., Suda, K., Fotiadis, D., Engel, A., Meier, T., Matthey, U. \& Dimroth, P. (2001) Bacterial $\mathrm{Na}^{+}$-ATP synthase has an undecameric rotor. EMBO Reports, 2, 229-233.

Thalhammer, S., Stark, R.W., Muller, S., Wienberg, J. \& Heckl, W.M. (1997) The atomic force microscope as a new microdissecting tool for the generation of genetic probes. J. Struct. Biol. 119, 232-237.

Van der Werf, K.O., Putman, C.A.J., De Grooth, B.G., Segerink, F.B., Schipper, E.H., Van Hulst, N.F. \& Greve, J. (1993) Compact stand-alone atomic force microscope. Rev. Sci. Instrum. 64, 2892-2897.

Viani, M.B., Pietrasanta, L.I., Thompson, J.B., Chand, A., Gebeshuber, I.C., Kindt, J.H., Richter, M., Hansma, H.G. \& Hansma, P.K. (2000) Probing protein-protein interactions in real time. Nature Struct. Biol. 7, 644-647.

Wallace, M.I., Molloy, J.E. \& Trentham, D.R. (2003) Combined single-molecule force and fluorescence measurements for biology. J. Biol. 2, 4.1-4.4.5.

Weiss, S. (1999) Fluorescence spectroscopy of single biomolecules. Science, 283, 1676-1683.

Wolford, D.J., Scoot, B.A., Reimer, J.A. \& Bradley, J.A. (1983) Efficient visible luminescence from hydrogenated amorphous silicon. Physica B, 117-118, 920-922.

Wong, S.S., Joselevich, E., Woolley, A.T., Cheung, C.L. \& Lieber, C.M. (1998) Covalently functionalized nanotubes as nanometre-sized probes in chemistry and biology. Nature, 394, 52-55. 